\title{
Generation of Pulsed Electron Beams by Simple Cold Cathode Plasma Guns
}

\author{
B. WERNSMAN, H. F. RANEA-SANDOVAL, J. J. ROCCA, AND H. MANCINI
}

\begin{abstract}
Electron-beam pulses of current up to $20 \mathrm{~A}$ have been generated using glow discharge electron guns of simple construction. Beam pulses of 1-20 $\mu$ s long at energies between 2 and $15 \mathrm{keV}$ have. been created at helium pressures between 1 and 5 torr. Results obtained using 3-cm diameter aluminum cathodes of two different geometries are discussed. As an example of the use of these electron guns for laser excitation, we have excited an He-Zn metal vapor mixture. Three new infrared laser transitions in $\mathrm{ZnII}$ are reported.
\end{abstract}

$\mathrm{P}$ REVIOUSLY WE OBTAINED dc electron-beam currents up to $1 \mathrm{~A}$ using glow discharge electron guns [1]. Here we report the pulsed operation of electron guns of two different cold cathode geometries at currents one order of magnitude above those obtained for dc operation. The generation of electron beams in a gas environment can find applications in laser excitation [2] and material processing [3]. The electron guns are of very simple construction; each consisting of an aluminum cathode $3 \mathrm{~cm}$ in diameter surrounded by an insulating ceramic tube. The oxide layer covering the surface of the aluminum cathode provides a high secondary electron emission coefficient following the bombardment by ions and fast neutral atoms [1]. This allows for high electron-beam generation efficiencies.

The geometry of one of the cathodes is the same as the one previously used for dc electron-beam generation. It is shown in Fig. 1(a). The geometry and position of the anode is not too critical to the operation of the gun. In our experiment, the metallic vacuum chamber is used as the anode for the glow discharge. When the discharge is operated in helium at a pressure of a few torr, a large cathode fall develops. Most of the voltage drop applied between the electrodes occurs in this region adjacent to the cathode and the rest of the discharge is a nearly field free negative glow. Consequently, practically all of the electron acceleration occurs in the cathode fall region. If the anode is placed at a distance from the cathode several times larger than the cathode fall thickness, the shape of

Manuscript received December 19, 1985; revised February 25, 1986. This work was supported in part by the U.S. Air Force, the National Science Foundation under Grant ECS-8404727, and the NSF-CONICET (United States-Argentina) International Program under Grant INT8402914. J. Rocca received support from an NSF Presidential Young Investigator Award.

B. Wernsman and J. J. Rocca are with the Electrical Engineering Department, Colorado State University, Fort Collins, CO 80523.

H. F. Ranea-Sandoval is on leave from CIOp (CIC-BA) Optical Research Center, Rep. Argentina.

H. Mancini is with CEILAP (CITEFA-CONICET), Rep. Argentina.

IEEE Log Number 8609595.

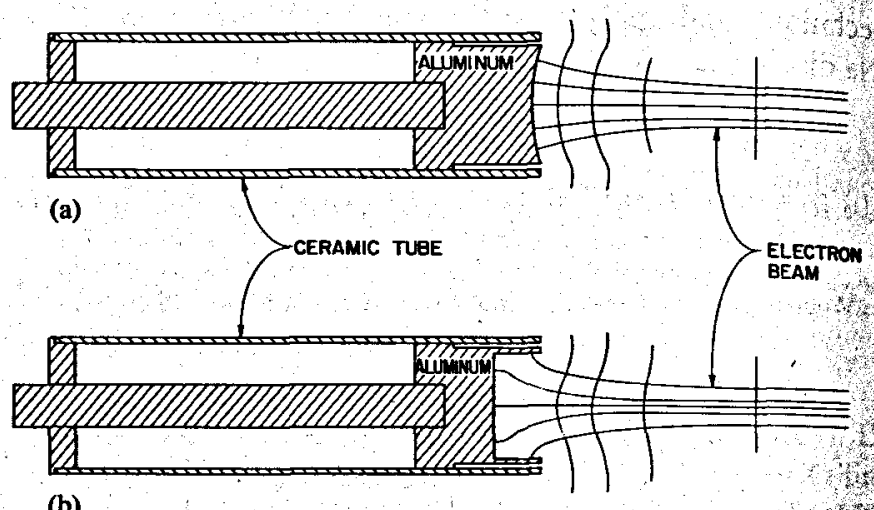

(b)

Fig. 1. Schematic representation of the electron guns. (a) and (b) show two different cathode geometries. The radius of curvature of the cathode front surface in (a) is $6 \mathrm{~cm}$.

the electric-field lines in the cathode dark space is mainly dependent on the cathode geometry. Consequently, the electron beam can be focused by tailoring the cathode shape. In the gun of Fig. 1(a), electron-beam focusing is achieved by making the cathode surface concave [3].

The second cathode geometry used in our experiments is shown in Fig. 1(b). It consists of an aluminum cylindrical cavity $3 \mathrm{~cm}$ in diameter and $1 \mathrm{~cm}$ in depth. Electron emission is achieved following ion bombardment of the inner walls of the cylindrical cavity. The cavity shapes the electric-field lines as indicated in Fig. 1(b). The emitted electrons are accelerated along the field lines to form the well collimated beam shown in Fig. 2 .

With this cathode geometry, good electron-beam focusing is achieved at pressures between 1 and 3 torr. At lower pressure, the cathode fall region broadens; the equipotential surfaces become flatter, and the focusing effect decreases. At pressures above 4 torr, the equipotential lines penetrate too deeply inside the cavity and follow the contour of the metal walls. This decreases electron-beam extraction, and the discharge collapses into a hollow cathode discharge.

The experiments were conducted in a stainless steel vacuum chamber which had a 4-in diameter in the shape of a cylindrical cross. This setup is illustrated in Fig. 3. The chamber was grounded and acted as the anode for the glow discharge. The cathode was pulsed negatively up to $15 \mathrm{kV}$ by connecting a $25-\mathrm{nF}$ capacitor through a triggered spark-gap or alternatively by using a pulse transformer providing voltages up to $5 \mathrm{kV}$. In the former case, a $10-\Omega$ resistor was used in series with the discharge. The 


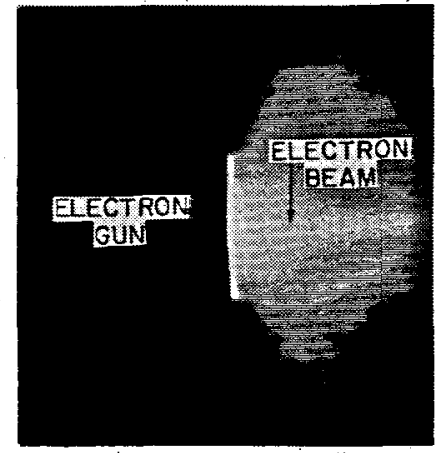

Fig. 2. Electron beam generated with the cathode of Fig. 1(b). Total current $3.9 \mathrm{~A}$; discharge voltage $4 \mathrm{kV}$; He pressure 2.4 torr; pulsewidth 50 $\mu$; pulse repetition rate $\cong 670 \mathrm{~Hz}$.

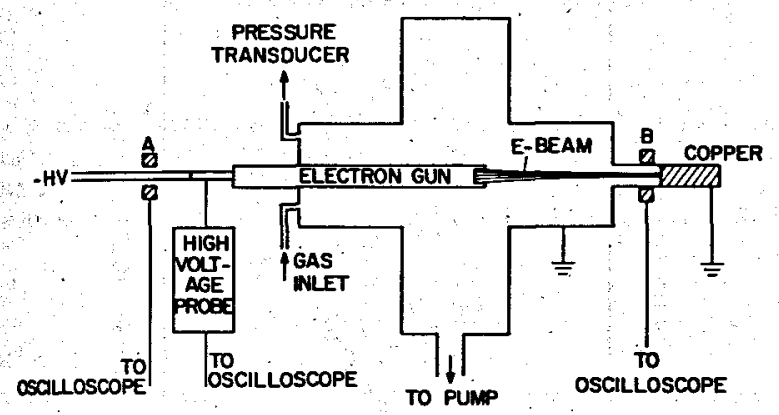

Fig. 3. Experimental setup used in the electron-beam generation experiments. $A$ and $B$ represent the pulse tranisformers used to measure the total discharge current and electron-beam current, respectively. Pulse transformer $B$ is mounted on an insulating cylinder.

total discharge current was measured by monitoring the cathode current with a pulse transformer. ${ }^{1}$ The discharge voltage was measured using a commercially available high voltage divider probe. ${ }^{2}$ The electron-beam current was estimated by shooting the electron beam through a second pulse transformer as indicated in Fig. 3. The current probe was mounted around an insulating cylinder placed between i grounded copper collector and the rest of the vacuum chamber.

The variation of the total glow discharge and electronbeam currents as a function of helium pressure for both cathode geometries is shown in Fig. 4. To obtain these data, approximately 30 mtorr of oxygen was added to the discharge to maintain a stable oxide layer and to ensure efficient electron emission at high repetition rates. When the measurements were repeated without the addition of oxygen, slightly lower ( 20 percent) currents were obtained. Fig. 4(a) corresponds to the electron gun geometry represented in Fig. 1(a). The discharge voltage was 13 ti and remained approximately constant for all values of the pressure between 1 and 4 torr. The electron-beam curtent constitutes approximately 80 percent of the total discharge current. This is in agreement with the values of dc electron-beam generation efficiencies obtained from cabrimetric measurements [1].

Fig. 4(b) corresponds to the electron gun having the athode geometry shown in Fig. 1(b). Electron-beam cur-

'Pearson Electronics, Model 110 current monitor, $0.1 \mathrm{~V} / \mathrm{A}$ output. Teltronix Inc., Model 6015 high-voltage divider probe, $40 \mathrm{kV}, 4$-ns
tue time.

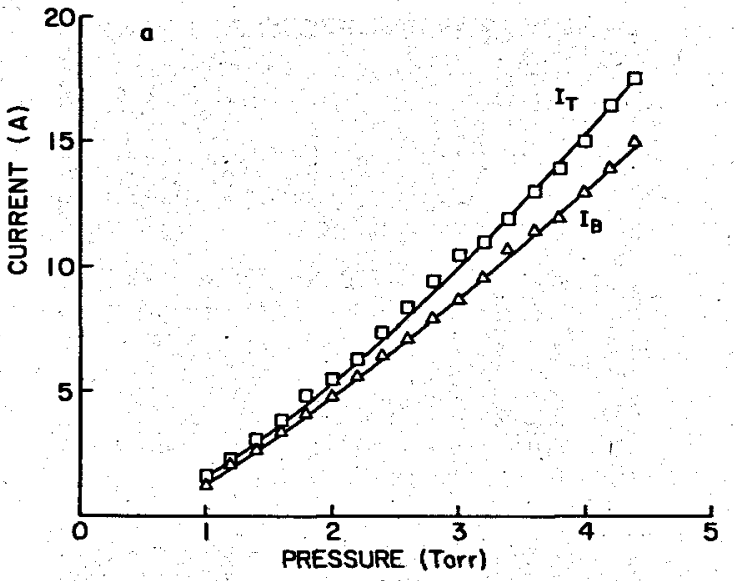

(a)

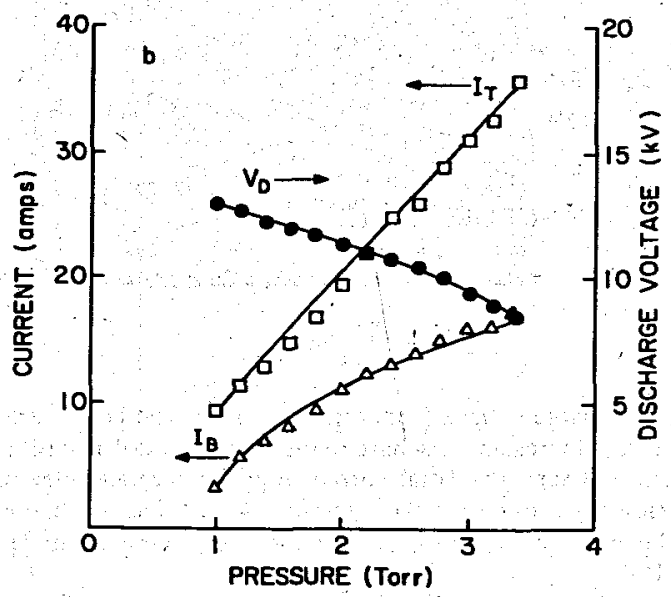

(b)

Fig. 4. Electron-beam current $I_{B}$, total discharge current $I_{T}$, and discharge voltage $V_{D}$ as a function of He pressure; 30 mtorr of $\mathrm{O}_{2}$ was added to the discharge. (a) and (b) correspond to the electron gun geometries of Fig. 1 (a) and (b), respectively. In (b), $V_{D}$ was constant.

rents up to 18 A were obtained. However, the electronbeam generation efficiency is smaller and approximately equal to $\mathbf{5 0}$ percent. The discharge voltage also shows a different behavior. It decreases as the pressure is increased as illustrated in Fig. 4(b). The different behavior of both cathodes might be due to the fact that, as the pressure is increased, the cathode of Fig. 1(b) tends to develop a hollow cathode behavior. A dense plasma is created within the cathode cavity causing a decrease in the discharge impedance. This argument is supported by the fact that if the pressure is further increased or the cathode is made deeper $(2 \mathrm{~cm})$ the glow will behave as a lower impedance hollow cathode discharge.

Fig. 5(a) shows the discharge voltage and corresponding electron-beam current pulse for the electron gun of Fig. 1(a) operating at 4 torr. Fig. 5(b) shows a simultaneous recording of the total discharge current and electron-beam pulse obtained with the same electron gun.

Fig. 6 illustrates the current pulses obtained with the electron gun geometry of Fig. 1(b) operating at 2.6 torr. The peak electron-beam current in this photograph is 14 $A$ for an applied voltage of $15 \mathrm{kV}$. By adding up to 0.1 


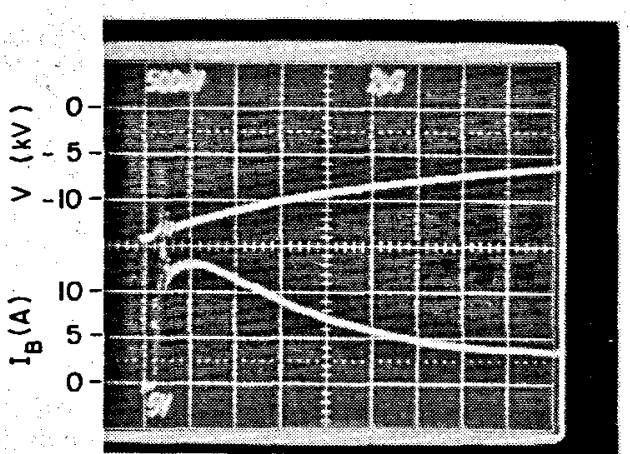

(a)

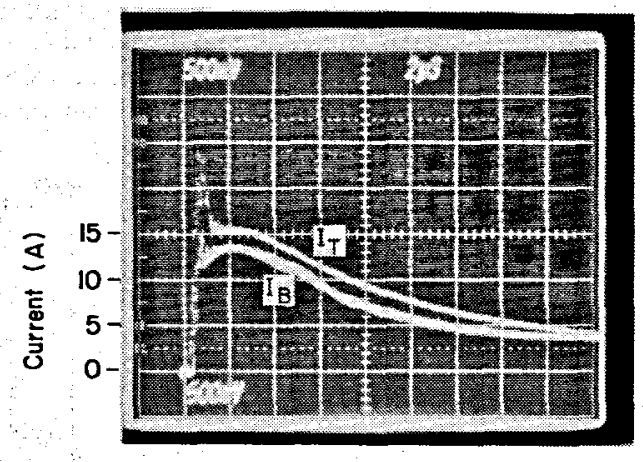

Time

(b)

Fig. 5. (a) Discharge voltage (upper trace) and electron-beam current pulse (lower trace). Increasing discharge voltage is in a downward direction. He pressure: 4 torr. (b) Total current (upper trace) and electron-beam current (lower trace) pulses. He pressure: 4 torr. The discharge voltage at peak beam current is $13 \mathrm{kV}$. The electron gun geometry is represented in Fig. 1(a).

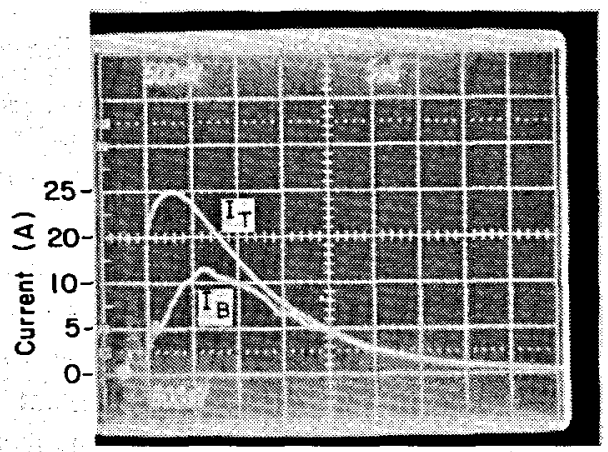

Time

Fig. 6. Discharge current (upper trace) and electron-beam current (lower trace) pulses. He pressure: 2.6 torr. Peak discharge voltage $15 \mathrm{kV}$. The electron gun geometry is represented in Fig. 1(b). Peak beam current is $14 \mathrm{~A}$.

torr of $\mathrm{O}_{2}$ to the discharge, stable 20-A beam current pulses were obtained.

In order to use these electron guns for laser excitation, it is useful to have an optical path through the axis. In this way, the volume of the plasma excited with the electron beam can be overlapped with the volume of an optical resonator [2]. For this purpose, we opened a hole $0.5 \mathrm{~cm}$ in diameter through the axis of the 3-cm-diameter electron guns described above. The hole was insulated with a quartz tube to maintain the high impedance operation of

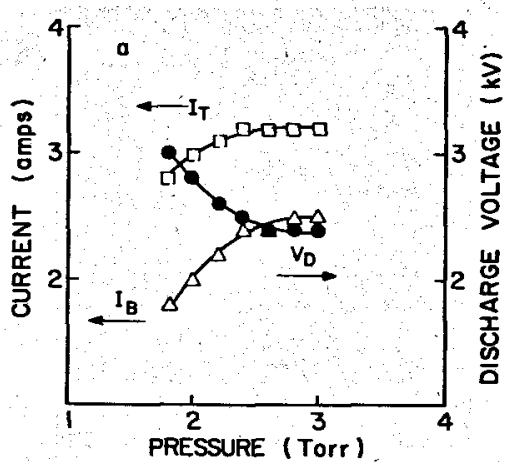

(a)

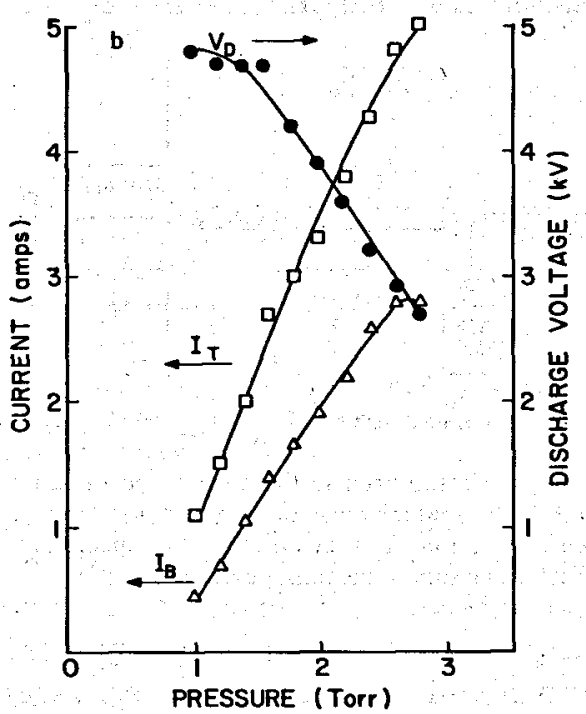

(b)

Fig. 7. Electron-beam current $I_{B}$ and total discharge current $I_{T}$ as a function of He pressure; 30 mtorr of $\mathrm{O}_{2}$ was added to the discharge. (a) and (b) correspond to the electron gun geometries of Fig. 1(a) and (b), trspectively, with the difference that they have a $0.5-\mathrm{cm}$ diameter hole in their axis.

the glow discharge. The axial hole, however, limits the maximum electron-beam current that can be obtained from these guns, since at high discharge power, the discharge tends to collapse into an arc. With the axial hole, the gun of Fig. 1(b) was found to give slightly higher beam currents as compared to the one of Fig. 1(a). Its operation was also more reliable.

These electron guns were excited with the output from a pulse transformer driven by a pulse-forming network giving rectangular voltage pulses of controllable duration. Fig. 7(a) and (b) shows the variation of the total discharge current and electron-beam current of these guns as a function of helium pressure for an applied voltage of $5 \mathrm{kV}$.

Fig. 8 shows the discharge voltage and corresponding total discharge current for a gun similar to that shown in Fig. 1(b) but having a $0.5-\mathrm{cm}$ diameter hole through its axis. Multiampere discharge current pulses can be ob tained at $1-\mathrm{kHz}$ repetition rates. We operated the electron gun at pulse rates up to $2 \mathrm{kHz}$ and at pulsewidths betwet? 5 and $100 \mu$ s.

As an example of the use of these electron guns for laser pumping, we excited an $\mathrm{He}-\mathrm{Zn}$ metal vapor mixture 


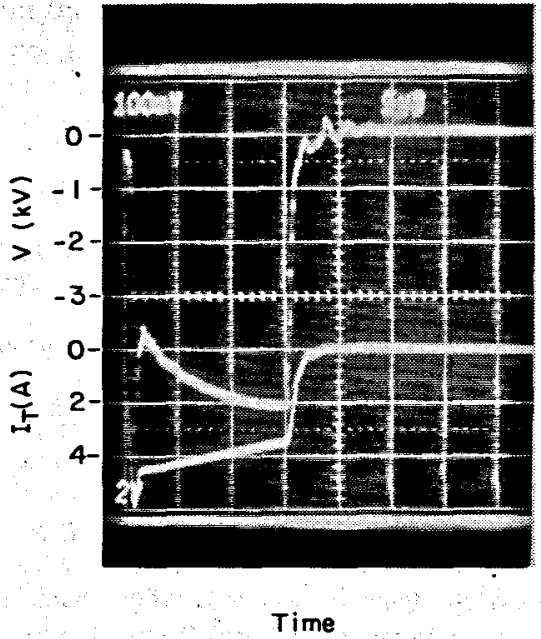

Fig. 8. Electron-beam discharge voltage (upper trace) and glow discharge current pulse (lower trace). Increasing discharge voltage is in the downward direction. The He pressure was 4 torr. Electron gun geometry corresponds to that of Fig. 1(b) with a $0.5-\mathrm{cm}$ diameter hole in its axis.

TABLE I

NEW ZnII LASER LINES

\begin{tabular}{|c|c|}
\hline Bavelength $(\mu \mathrm{m})$ & Transition Assignment \\
\hline 1.83133 & $6 \mathrm{p}{ }^{2} \mathrm{P}_{3 / 2}^{0}-6 \mathrm{~s}^{2} \mathrm{~s}_{1 / 2}$ \\
\hline 2.15003 & $5 p{ }^{2} P_{3 / 2}^{0}-4 d^{2} D_{5 / 2}$ \\
\hline 2.24401 & $5 p^{2} \mathrm{P}_{1 / 2}^{0}-4 \mathrm{~d}^{2} \mathrm{D}_{3 / 2}$ \\
\hline
\end{tabular}

and obtained laser action in three new ZnII laser transitions. The laser setup was similar to that previously used to excite CW metal vapor lasers using a dc electron beam [2]. The electron beam was injected into a stainless steel plasma tube $1 \mathrm{~cm}$ in diameter and $100 \mathrm{~cm}$ long. The tube was maintained at $600^{\circ} \mathrm{C}$ using an external heater, and Zn vapor was introduced by heating a reservoir to $450^{\circ} \mathrm{C}$. An electromagnet surrounding the plasma tube provided an axial magnetic field of up to $2.5 \mathrm{kG}$ to guide the electon beam. At a helium pressure of 1.5 torr, three new infrared laser transitions were observed in ZnII when a resonator was constructed using two, $2-m$ radius-of-curvature mirrors having high reflectivity in the $1.5-2-\mu \mathrm{m}$ pectral region. The transition wavelengths and level assignments are listed in Table $I$.

A $90-\mu$ s electron-beam discharge current pulse and the corresponding laser output at $1.83133 \mu \mathrm{m}$ as observed through a monochromator are shown in Fig. 9. Laser action occurs during the entire electron-beam current pulse. The variation of the laser output power of the 1.83133in $\left(6 p^{2} P_{3 / 2}^{0}-6 s^{2} S_{1 / 2}\right)$ transition as a function of discharge current is shown in Fig. 10. The total discharge arent threshold for oscillation of this line was found to be $600 \mathrm{~mA}$. The laser upper levels are excited mainly by charge transfer collisions between helium ions and neutral inc atoms.

The presence of the small amount of oxygen added into

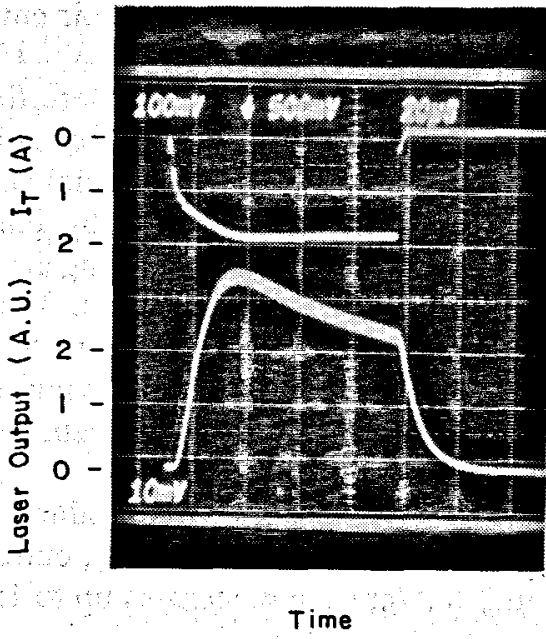

Fig. 9. Discharge current (upper trace) and ZnIl laser output at 1.83133 $\mu \mathrm{m}$ (lower trace) as a function of time. Discharge voltage $2 \mathrm{kV}$; pulse repetition rate $\cong 670 \mathrm{~Hz}$. The time scale is $20 \mu \mathrm{s} / \mathrm{div}$.

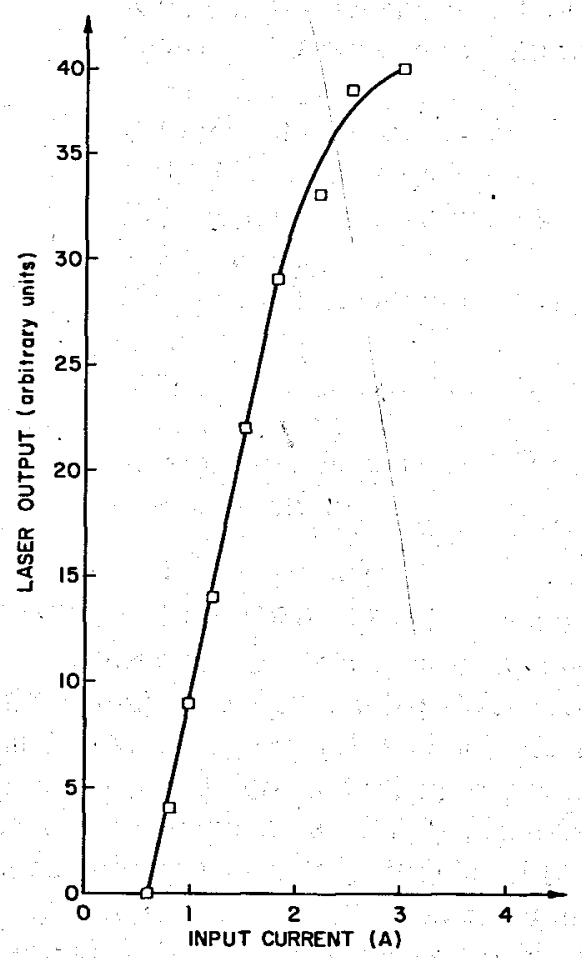

Fig. 10. Laser output power of the $1.83133-\mu \mathrm{m}$ line as a function of electron-beam discharge current. Zn reservoir temperature was $500^{\circ} \mathrm{C}$. The average $\mathrm{He}$ pressure in the plasma tube was 1.5 torr.

the electron gun region was not observed to have any detrimental effect on the laser output. This result is in agreement with a previous experiment in which we used the same electron guns to obtain laser oscillation in $\mathrm{CdI}$ and CdII [4]. In the excitation of other laser transitions, such as the excitation of ultraviolet metal vapor lasers, the presence of oxygen in the active medium might present a more serious problem. In this case, sintered metal-oxide cathode materials (e.g., molybdenum-aluminum oxide) can be used. These materials have a high secondary emission coefficient and are able to operate in a pure noble gas 
atmosphere without a degradation of their emissive characteristics [3].

The pulsed electron guns described here find an interesting application in the excitation of recombination lasers. The beam electrons can provide efficient ionization, and cold secondary electrons created by ionization can readily recombine to populate high-lying levels through three-body electron-ion recombinations. We have used the pulsed electron beams described above to obtain laser action in three transitions of CdI following electron-ion recombination. These results will be discussed in another publication [4].

In summary, we demonstrated the production of pulsed electron beams with currents up to $20 \mathrm{~A}$, current densities of more than $2.5 \mathrm{~A} / \mathrm{cm}^{2}$, and energies up to $15 \mathrm{keV}$ using electron guns of simple construction having aluminum cathodes. Its application to the excitation of metal vapor lasers was also demonstrated.

\section{REFERENCES}

[1] J. J. Rocca, J. Meyer, Z. Yu, M. Farrell, and G. J. Collins, "Multikilowatt electron guns for CW laser excitation," Appl. Phys. Lets vol. 41, pp. 811-813, Nov. 1, 1982.

[2] J. J. Rocca, J. Meyer, and G. J. Collins, "1-W zinc ion laser," Appl. Phys. Lett., vol. 43, pp. 37-39, July 1, 1983.

[3] J. J. Rocca, J. Meyer, M. Farrell, and G. J. Collins, “Glow discharge created electron beams: Cathode materials, electron gun designs, and technological applications,"'J. Appl. Phys., vol. 56, pp. 790-797, Aug. 1, 1984.

[4] J. J. Rocca, H. Mancini, and B. Wernsman, "CD recombination laser in a plasma generated by an electron beam," IEEE J. Quantum Elec. tron., vol. QE-22, pp. 509-512, Apr. 1986. 